\title{
ALMA and VLA observations of emission from the environment of Sgr A*
}

\author{
F. Yusef-Zadeh, ${ }^{1 \star}$ R. Schödel ${ }^{2}$ M. Wardle ${ }^{3}$ H. Bushouse ${ }^{4}$ W. Cotton ${ }^{5}$ \\ M. J. Royster ${ }^{1}$ D. Kunneriath ${ }^{2}$ D. A. Roberts ${ }^{1}$ E. Gallego-Cano ${ }^{2}$ \\ ${ }^{1}$ Department of Physics and Astronomy Northwestern University, Evanston, IL 60208, US \\ ${ }^{2}$ Instituto de Astfisica de Andalucia (CSIC), Glorieta de la Astronomia S/N, 18008 Granada, Spain \\ ${ }^{3}$ Department of Physics and Astronomy and Research Centre for Astronomy, Astrophysics \\ ES Astrophotonics, Macquarie University, Sydney NSW 2109, Australia \\ ${ }^{4}$ Space Telescope Science Institute, Baltimore, MD 21218 \\ ${ }^{5}$ National Radio Astronomy Observatory, Charlottesville, VA 22903
}

16 March 2022

\begin{abstract}
We present 44 and $226 \mathrm{GHz}$ observations of the Galactic center within $20^{\prime \prime}$ of Sgr A*. Millimeter continuum emission at $226 \mathrm{GHz}$ is detected from eight stars that have previously been identified at near-IR and radio wavelengths. We also detect a $5.8 \mathrm{mJy}$ source at $226 \mathrm{GHz}$ coincident with the magnetar SGR J1745-29 located 2.39" SE of Sgr A* and identify a new $2.5^{\prime \prime} \times 1.5^{\prime \prime}$ halo of mm emission centered on Sgr A*. The X-ray emission from this halo has been detected previously and is interpreted in terms of a radiatively inefficient accretion flow. The mm halo surrounds an EW linear feature which appears to arise from Sgr $\mathrm{A}^{*}$ and coincides with the diffuse X-ray emission and a minimum in the near-IR extinction. We argue that the millimeter emission is produced by synchrotron emission from relativistic electrons in equipartition with a $\sim 1.5 \mathrm{mG}$ magnetic field. The origin of these is unclear but its coexistence with hot gas supports scenarios in which the gas is produced by the interaction of winds either from the fast moving S-stars, the photo-evaporation of low-mass YSO disks or by a jet-driven outflow from Sgr A*. The spatial anti-correlation of the Xray, radio and $\mathrm{mm}$ emission from the halo and the low near-IR extinction provides compelling evidence for an outflow sweeping up the interstellar material, creating a dust cavity within $2^{\prime \prime}$ of Sgr A*. Finally, the radio and $\mathrm{mm}$ counterparts to eight near-IR identified stars within $\sim 10^{\prime \prime}$ of Sgr $\mathrm{A}^{*}$ provide accurate astrometry to determine the positional shift between the peak emission at 44 and $226 \mathrm{GHz}$.
\end{abstract}

Key words: accretion, accretion disk - black hole physics - Galaxy: centre

\section{INTRODUCTION}

The nuclear region of our Galaxy coincides with a stellar cluster consisting of an evolved population and a young population of OB and WR stars (Paumard et al. 2006; Lu et al. 2009) centered on the $4 \times 10^{6} M_{\odot}$ black hole Sgr A* (Reid and Brunthaler 2004; Genzel et al. 2010; Schödel et al. 2014; Boehle et al. 2016; Gillsessen et al. 2016). Until recently, the young massive stars within 0.5 pc of Sgr A* could only be identified and studied employing

^ E-mail: zadeh@northwestern.edu (FYZ)

(C) 2015 The Authors 
adaptive optics in the near-IR. Recent high-resolution radio continuum observations detected 318 compact radio sources within the inner $30^{\prime \prime}$ of Sgr A*. The comparison of radio and near-IR data indicate that at least 45 of the compact radio sources coincide with known sources, many of which are massive stars, identified at $K_{S}$ and $L^{\prime}$ bands in the near-IR (Yusef-Zadeh et al. 2016).

Thermal radio emission from a mass-losing star arises from spherically-symmetric, wind of fully-ionized gas expanding at its terminal velocity (e.g., Panagia \& Felli 1975). Stellar thermal emission at radio wavelengths could also arise from the photospheres of evolved stars in the nuclear cluster. One way to distinguish between these two different populations is to determine their radio spectral index $\alpha$ where the flux density $\mathrm{S}_{v} \propto v^{-\alpha}$. Photospheric emission has an inverted spectrum with $\alpha \sim 2$ whereas ionized stellar winds from young massive stars typically have a spectrum with $\alpha \sim 0.6$ (Panagia 1973). Previous detection of radio emission from near-IR identified stars in the inner $10^{\prime \prime}$ of Sgr A* (Yusef-Zadeh et al. 2015a) had a limited frequency coverage between 34 and $44 \mathrm{GHz}$, thus, it was not possible to accurately determine the spectral index. The present $226 \mathrm{GHz}$ and $44 \mathrm{GHz}$ observations present an opportunity to remedy this by determining the spectrum of radio emission and distinguishing between members of the evolved cluster and massive stars in the young cluster.

To measure orbits of stars around Sgr A*, it is necessary to tie radio and near-IR data into astrometric reference frames. The positions of radio stars can provide precise astrometry relative to Sgr $\mathrm{A}^{*}$. The detection of stars at millimeter $(\mathrm{mm})$ wavelengths opens a new window for astrometric calibration as well as examining if there is a shift in the peak position of $\mathrm{Sgr} \mathrm{A}^{*}$ between radio and $\mathrm{mm}$ wavelengths as might be expected, for example if $\mathrm{mm}$ emission from a jet was present (Markoff et al. 2007)

Here we present simultaneous ALMA, VLA and VLT observations of the Galactic center and determine the spectral index between 44 and $226 \mathrm{GHz}$ of eight stellar sources identified at $\mathrm{H}(1.63 \mu \mathrm{m})$ band and two nonthermal sources Sgr A* and the magnetar SGR J1745-29. In addition, we determine the peak position of Sgr A* at $226 \mathrm{GHz}$ by registering the accurate positions of near-IR identified stars detected at 44 and $226 \mathrm{GHz}$ and then search for any shifts in position of Sgr A* at 44 and $226 \mathrm{GHz}$. Finally, the observations presented here show sub-structures associated with Sgr $\mathrm{A}^{*}$ and a network of narrow features at radio and $\mathrm{mm}$. We detect a halo of mm emission from the inner $2^{\prime \prime}$ of Sgr A* which coincides with low near-IR extinction. The size of this halo is similar to that detected at X-rays. We suggest that the outflow from the ionized winds of the S stars orbiting Sgr A* are responsible for this emission. We report tentative detection of faint narrow fibrils of radio and $\mathrm{mm}$ emission. We interpret these striking features as arising from the interaction of radial outflows from the Galactic center and the atmospheres of mass-losing stars.

\section{OBSERVATIONS AND DATA REDUCTION}

\subsection{Radio and Millimeter Data}

The ALMA, VLA ${ }^{1}$, and VLT observations were carried out as part of a multi-wavelength observing campaign to monitor the flux variability of Sgr A*. This campaign was led by Spitzer and Chandra, and radio and $\mathrm{mm}$ observations were obtained as part of the director's discretionary time given to us to join the observing campaign. Titan was initially used in ALMA observations (project code 2015.A.00021.S.) as the flux calibrator and NRAO 530 was observed periodically to correct for any changes in phase and amplitude as a function of time. The spectral windows were centered at roughly: $216.2 \mathrm{GHz}, 218.0 \mathrm{GHz}, 231.9 \mathrm{GHz}$, and 233.7 GHz. Editing and calibration of the data were carried out with OBIT (Cotton 2008) before all the spectral windows were averaged prior to constructing final images. Observations were made on July 12 and 18, 2016 and the images were combined after scaling the variable flux of Sgr A* during the two epochs of observations.

We carried out VLA B-array observations (program 16A-419) in the Q (7mm) and Ka (9mm) bands on July 12 and 18, 2016 at 44 and $34 \mathrm{GHz}$, respectively. We used the 3-bit system, which provided full polarization in 4 basebands, each $2 \mathrm{GHz}$ wide. Each subband was made up of 64 channels and channels were $2 \mathrm{MHz}$ wide. We used 3C286 to calibrate the flux density scale and used 3C286 and J1733-1304 (aka NRAO530) to calibrate the bandpass and J1744-3116 to calibrate the complex gains. A phase and amplitude self-calibration procedure was applied to all data using the bright radio source Sgr A*. We used OBIT (Cotton 2008) and CASA to construct radio and $\mathrm{mm}$ images. The positions of radio stars are determined with respect to the absolute position of Sgr A*. The Ka band data were compromised by bad weather conditions, thus we do not present those observations.

1 the Karl G. Jansky Very Large Array (VLA) of the National Radio Astronomy Observatory is a facility of the National Science Foundation, operated under a cooperative agreement by Associated Universities, Inc. 


\section{$2.2 \quad$ VLT Data}

NACO/VLT was used as part of a coordinated observing campaign, as described above. Ten hours of observations were granted but the observations suffered from bad seeing conditions, with the coherence time being mostly $\tau_{0} \lesssim 3 \mathrm{~ms}$ and the isoplanatic angle $\theta_{0} \approx 1$ " arcsec. Consequently, the Adaptive Optics (AO) performance was mostly poor, with the exception of a few short intervals of good conditions. As is standard for NACO AO observations of the GC, the loop of the AO system was closed on the $K_{s} \approx 7$ supergiant IRS 7 , located about 5.5" north of Sgr A*. For this work we use the best data set, which was obtained on 2016 July 12 during UT 04:26 to 04:58. It consists of observations in the $H$-band with the S27 camera. The exposure time was set to $3 \mathrm{~s}$. We used NACO's cube-mode and obtained 26 sets of 21 exposures each, amounting to a total integration time of $1638 \mathrm{~s}$. Data reduction was standard (sky subtraction, flat-field correction, dead-pixel interpolation) and all the reduced exposures were aligned via the centroid of the star IRS16 C and mean-combined. Stellar positions and fluxes were extracted with the StarFinder package (Diolaiti et al. 2000). We also present the extinction map of the inner 30" derived from star counts in the near-IR, details of which are explained in Schödel et al. (2010). Foreground stars were removed prior to creating the extinction map, which thus provides a good measure of the column density of the clouds in the Galactic center.

\section{RESULTS}

\subsection{A 2.5" $\times 1.5^{\prime \prime}$ Emission Halo Centered on Sgr A*}

Figure 1a shows the inner $17^{\prime \prime} \times 18^{\prime \prime}$ of $\mathrm{Sgr} \mathrm{A}^{*}$ at $225 \mathrm{GHz}$ where the brightest portion of the mini-spiral structure associated with the three-arms of the minispiral (Sgr A West) are detected. The N and E arms and the bar to the south of Sgr A* are prominent. The region within a few arcseconds of Sgr A*, outlined schematically by a dashed semi-circle, shows a number of new structures. One is a diffuse halo structure with an elliptical appearance within $2.5^{\prime \prime} \times 1.5^{\prime \prime}$ of Sgr A* with mean flux density of $\sim 0.2-0.5 \mathrm{mJy}$ beam $^{-1}$. The integrated flux is $81 \mathrm{mJy}$ over an area of 9.7 square arcsecond. This diffuse structure has an X-ray counterpart (Wang et al. 2013). Figure $1 \mathrm{~b}$ shows a composite image of $\mathrm{mm}$ and X-ray emitting gas where we note the diffuse X-ray emission is coincident with the mm halo centered on Sgr A*. The NS elongated X-ray structure G359.945-0.044 about 10" NW of Sgr $\mathrm{A}^{*}$ is a pulsar wind nebula candidate (Wang et al. 2013). Figure 1c shows the composite image of the extinction in the near-IR and the mm emission. The extinction value in the Ks band $(2.17 \mu \mathrm{m})$ ranges between 2.3 and 3.2 (Schödel et al. 2010). We note the halo structure has the lowest extinction $\sim 2.4$ magnitudes compared to the high extinction of $\sim 2.9$ magnitudes surrounding it. Dark dashed lines to the south of Sgr A* outline the elliptical halo structure bounded by the bar to the south of $\mathrm{Sgr} \mathrm{A}^{*}$. We also note the high extinction associated with the $\mathrm{N}$ and $\mathrm{E}$ arms and the bar of the mini-spiral (Schödel et al. 2010). The extinction map of the mini-spiral shows clearly the complex nature of cold and dense gas that is externally photoionized by the Galactic center radiation field to create the minispiral.

We note a bow-shock-like structure with an extent of $\sim 2^{\prime \prime}$ to the NE of Sgr A*. The typical surface brightness of this feature is about $0.2 \mathrm{mJy}_{\text {beam }}^{-1}$ at $226 \mathrm{GHz}$. Finally, a EW mm ridge of emission protrudes from Sgr $A^{*}$. This ridge appears to extend further to the west for several arcseconds before it merges with the continuum emission from the mini-spiral.

A close-up view of the inner $6^{\prime \prime} \times 4^{\prime \prime}$ of $\mathrm{Sgr} \mathrm{A}^{*}$ is shown in Figure 2a where contours of mm emission are superimposed on 1.5-7 keV X-ray emission. The extended X-ray emission was identified by Wang et al. (2013). This image shows a spatial correlation between $\mathrm{mm}$ and X-ray emission. The diffuse X-ray and mm emission from the halo coincide with a region of low extinction. The mm contours show an elliptically-shaped $2.5^{\prime \prime} \times 1.5^{\prime \prime}$ halo of diffuse emission similar to the X-ray morphology. The anti-correlation of high emission and low extinction suggest clearly that the low extinction is caused by an outflow. Figure $2 \mathrm{~b}$ shows the close-up view of $226 \mathrm{GHz}$ emission from Sgr $A^{*}$ and its vicinity. The elongated EW structure or the linear ridge appears to be associated with Sgr $A^{*}$. The bow shock feature lies to the eastern edge of the diffuse halo. The bow-shock may indicate where an outflow from Sgr A* interacts with the ISM in the immediate vicinity of Sgr A*." We also note compact sources that coincide with stellar sources, as described below. 


\subsection{Compact Stellar Sources at $226 \mathrm{GHz}$}

Ten compact sources at 44 and $226 \mathrm{GHz}$ are identified within the inner $10^{\prime \prime}$ of Sgr A*. These sources, labeled on Figures $2 \mathrm{~b}$ and $3 \mathrm{a}, \mathrm{b}$, are used to astrometrically register the $\mathrm{mm}$ and near-IR images. As all radio, mm and near-IR data are taken at the same epoch, we identified radio and mm stars from the first ALMA epoch 2016.54 by comparing all three images. ALMA observations detect eight near-IR identified stellar sources at $226 \mathrm{GHz}$. These stars also have counterparts at $44 \mathrm{GHz}$. Tables 1 and 2 list Gaussian-fitted positions of 10 radio and mm sources distributed within the inner 10" of Sgr A* at 44 and $226 \mathrm{GHz}$. Entries in the columns of Tables 1 and 2 give the source name at 44 and $226 \mathrm{GHz}$, alternative names in the literature, the RA and Dec, the angular distance from Sgr A* in increasing order, positional accuracy, the size of the source, the peak and integrated intensities. Column 9 of Table 1 gives the spectral index of individual sources between 44 and $226 \mathrm{GHz}$. The last column provides the comments on individual sources. The positional accuracy is determined from quadrature sum of errors of the right ascension and declination values from 2D Gaussian fits without including absolute astrometric errors. Because the second epoch of ALMA data had a higher spatial resolution, we compared this epoch with the first epoch of VLA observation at $44 \mathrm{GHz}$ to determine the spectral index of all sources except Sgr A*. The spectral index of Sgr $A^{*}$ is estimated from the same epoch data sets. The positions and the sizes of radio sources are determined from background-subtracted Gaussian fit to the individual radio sources.

We note the radio source associated with IRS 21 has a mm counterpart. This young stellar source (SanchezBermudez et al. 2014) is comprised of five radio components (Yusef-Zadeh et al. 2014) but only one stellar source is identified at H-band. The radio and infrared properties are similar to those of IRS 13N and IRS 13E suggesting that the radio emission arises from the disks of massive YSO candidates in this cluster (Yusef-Zadeh et al. 2015b). We also detect mm emission from the Galactic center magnetar, SGR J1745-29 (Kennea et al. 2013; Shannon and Johnston 2013; Torne et al. 2017). This source was in its quiescent phase before it was identified as an X-ray outburst (Kennea et al. 2013). SGR J1745-29 is the closest known pulsar to Sgr A* located 2.4" from Sgr A*. The detection of a compact radio source was reported at $\alpha, \delta(J 2000)=17^{h} 45^{m} 40^{s} .16795 \pm 0.00002-29^{\circ} 00^{\prime} 29^{\prime \prime} .74908 \pm$ 0.00064 at $44.6 \mathrm{GHz}$ on 2014 February 21 (Yusef-Zadeh et al. 2014). Our $44 \mathrm{GHz}$ image shows a plume-like structure to the north of the magnetar. This plume-like feature with an extent of $0.2^{\prime \prime} \times 0.5^{\prime \prime}$ (width $\times$ length) widens to the north with a peak flux density $0.8 \mathrm{mJy}_{\text {beam }}{ }^{-1}$. It does not have a counterpart at $3.8 \mu \mathrm{m}$ (Eckart et al. 2013) and has no obvious counterpart at $226 \mathrm{GHz}$. We do not have sufficient data to determine its spectral index. Future polarization and spectral studies of this feature would determine its nature.

The spectral index of $\operatorname{Sgr} \mathrm{A}^{*} \alpha=0.56$ listed in Table 1 is steeper than previously determined from snapshot measurements (An et al. 2005). The magnetar has a relatively flat spectrum with $\alpha=-0.21$. The mm emission from the magnetar could be due to the combination of pulsed and diffuse shocked emission produced by the interaction of the pulsar outburst with the ISM (Yusef-Zadeh et al. 2016). The remaining eight sources are stellar, six of which have spectra consistent with ionized winds. Sources 7 and 8, which coincide with IRS 3 and IRS 7SW, respectively, have a steep optically thick spectrum consistent with photospheric radio emission. Alternative, these steep spectrum sources could also be generated by ionized winds of massive stars with or wind sources with a varying density gradient and geometry (Panagia \& Felli 1975). IRS 3 is the brightest and most extended $3.8 \mu \mathrm{m}$ Galactic center (Pott et al. 2008) stellar source. The asymmetric shape of the IRS 3 envelope may reflect tidal distortion by Sgr A* (Yusef-Zadeh et al. 2017).

\subsection{Search for Radio and $\mathrm{mm}$ Emission from the $\mathrm{S}$ stars}

There is a cluster of B dwarf stars associated with the S-star cluster within 1" of Sgr A* (Gillessen et al. 2009, 2016; Yelda et al. 2010, 2014). The detection of $\mathrm{S}$ stars in the radio and $\mathrm{mm}$ has been challenging because of the bright and variable source Sgr A* and its frequency dependent angular size due to interstellar scattering. To search for radio emission from stars within $1^{\prime \prime}$ of $\mathrm{Sgr} \mathrm{A}$, we first calculated the positions of the $\mathrm{S}$ stars at the epoch of the mm observation on 2016, July 16 by using orbital parameters derived from near-IR observations (Gillessen et al. 2016). Table 3 gives the positions of the S cluster members offset from Sgr A* in R.A. and Declination and their corresponding positional uncertainties at the epoch of 2016.54. The expected positions are indicated as crosses in three images, taken within a few days of each other, a $1.6 \mu \mathrm{m} \mathrm{H}$-band, $226 \mathrm{GHz}$ and $44 \mathrm{GHz}$ image, as shown in Figures 4a-c, respectively. The S stars are superimposed after astrometric corrections have been applied to the near-IR and $\mathrm{mm}$ images. The near-IR stellar sources coincide well with the predicted positions in Figure 4a. Two bright stellar sources S96 and S97 in Figure 4b coincide with IRS 16NE and IRS 16SE, respectively. There are also coincidences between some $\mathrm{S}$ stars, such as $\mathrm{S} 83$, and $\mathrm{mm}$ peaks in Figure $4 \mathrm{~b}$. However, there is extended $\mathrm{mm}$ emission, and it is not possible to localize mm counterparts to stellar sources, mainly because of the confusing 
compact and extended sources. Remarkably, we note a number of $44 \mathrm{GHz}$ sources throughout the inner $3^{\prime \prime}$ of Sgr $\mathrm{A}^{*}$, as shown in Figure 4c. In particular, S83 and S33 may have radio counterparts within the positional errors at $44 \mathrm{GHz}$. Similarly, S5 and S14 coincide with peak radio emission at a level of $0.15 \mathrm{mJy}^{\mathrm{beam}}{ }^{-1}$. Proper motion measurements in the radio are needed to establish that they are indeed counterparts to $\mathrm{S}$ stars.

We note a number of radio and mm peaks without stellar counterparts in the $\mathrm{H}$ band. Radio sources could have counterparts in the $\mathrm{L}^{\prime}$ band where a number of dusty sources or the so-called dusty S cluster objects (DSO/G2) have been detected (Eckart et al. 2014). However, we have neither the proper motion of DSO sources nor an $\mathrm{L}^{\prime}$ band image taken in the same epoch as the data presented here to confirm $3.8 \mu \mathrm{m}$ counterparts to radio peaks. A member of this class of objects detected in the $\mathrm{L}^{\prime}$ band is $\mathrm{G} 2$ that was the subject of intense observational campaigns as it passed extremely close to the central black hole (Gillessen et al. 2012; Witzel et al. 2014; Pfuhl et al. 2015; Valencia-Schneider et al. 2015). Given the low spatial resolution of our radio and mm observations, we could not search for radio counterparts to G2. The expected position of the G2 cloud is -85 and 80 mas from Sgr A* (Gillessen, private communication) which is smaller than the $222 \times 128$ mas synthesized beam at $44 \mathrm{GHz}$.

The compact radio and mm sources detected within $2^{\prime \prime}$ of $\mathrm{Sgr} \mathrm{A}^{*}$ with no stellar counterparts could be massive young stellar objects (YSOs), similar to radio counterparts of several members of the IRS 13N cluster (Eckart et al. 2013; Yusef-Zadeh et al. 2014, 2015b). In this interpretation, ionized gas is being photo-evaporated from the disks of YSOs by the UV radiation from young and massive stars located between 1 and 10" from Sgr A* (Yusef-Zadeh et al. 2014). Future proper motion and spectral measurements of radio and mm sources are critical to determine their nature.

\subsection{Dust Cavities near Sgr A*}

We also examined the physical relationship between the S stars and a dust cavity centered on Sgr A* that is present in the extinction map of Sgr A West, as shown in Figure 1c (Schödel et al. 2010). Figure 4d shows the inner $4^{\prime \prime} \times 3.5^{\prime \prime}$ of Sgr A* where the largest concentration of S stars is detected in the Sgr A* dust cavity. The dust cavity coincides with the elongated $\mathrm{mm}$ halo and excess diffuse X-ray and radio emission, as discussed earlier (see Figures 2a,b and 4b,c). In addition, the correlation of the dust cavity and a halo of enhanced emission at multiple wavelengths suggests an outflow has destroyed or swept the cold and dense material away from Sgr A*. This X-ray filled cavity with minimum extinction provides the strongest evidence for an outflow, the origin of which is discussed in the next section.

A larger view of the near-IR extinction map identifies regions of low and high columns of dust. Figure 5a,b show the inner $13^{\prime \prime}$ of $\mathrm{Sgr} \mathrm{A}^{*}$ delineating extinction clouds and $44 \mathrm{GHz}$ radio continuum emission, respectively. We note a cloud that lies along the northwestern extension of the $\mathrm{E}$ arm at $\sim-5^{\prime \prime}$ and $\sim 1^{\prime \prime} \mathrm{W}$ and $\mathrm{N}$ of Sgr $\mathrm{A}^{*}$, respectively. The anticorrelation of this dust cavity and a gap in the ionized gas strongly suggest that the dust cavity is associated with the ionized material.

The extinction map shown in Figure 5a (Schödel et al. 2010) reveals a second dust cavity $3^{\prime \prime}$ to the NE of the Sgr A* (drawn as a circle on Fig. 5a). The elongation and the position angles of the dust cavities are similar to the position angles of a tentative jet driven outflow from Sgr A* which was reported by Yusef-Zadeh et al. (2012). In addition, a number of resolved sources, X3, X7, F1, F2, F3, P1, P4 and the Sgr A East tower show elongated structures with similar position angles in radio and $3.8 \mu \mathrm{m}$ images (Muzic et al. 2007, 2010; Yusef-Zadeh et al. 2016). These elongated emitting features combined with elongated dust cavities, centered on $\operatorname{Sgr} \mathrm{A}^{*}$ and to the $\mathrm{NE}$ of Sgr A*, provide support for a common origin for the collimated outflow from Sgr A* at a position angle of $\sim 60^{\circ}$ (Schödel et al. 2007).

Another piece of evidence suggestive of an outflow from Sgr A* is an elongated feature from Sgr A* that curves to the SW for $\sim 5^{\prime \prime}$ and terminates in the mini-cavity. Figure $6 \mathrm{a}$,b show grayscale images of the inner $4^{\prime \prime} \times 6^{\prime \prime}$ of Sgr $\mathrm{A}^{*}$ and contours of $44 \mathrm{GHz}$ emission superimposed on a H-band image. The outline of the elongated edgebrightened balloon-shaped feature with a $4^{\prime \prime} \times 1^{\prime \prime}$ extent, is drawn on Figure 6a. The northern arc-like structure of this balloon-shaped structure, as shown in Wardle and Yusef-Zadeh (1992), coincides with a blob known as "epsilon" (Yusef-Zadeh et al. 1990) which has been detected in earlier low spatial resolutions at $15 \mathrm{GHz}$ (Zhao et al. 1991). The new image shows clearly that the blobs are extended and continue to form a balloon in the direction of the mini-cavity. A balloon-shaped structure to the SW and a dust cavity to the NE of Sgr A* suggest that these features are related. The proper motion measurement of the $\epsilon$ blob shows high velocity ionized gas moving away from Sgr A* to the SW (Zhao et al. 2009). This morphological and kinematic information suggests that the elongated features to the NE and SW are consistent with the outflow interpretation from Sgr A*. Future 
high resolution proper motion, polarization and spectral index measurements of the ridge will provide additional constraints on the claim that this feature is physically associated with Sgr A*.

\subsection{Near-IR and Millimeter Astrometry}

Given the accurate position of Sgr A* at radio wavelength (Reid et al. 1999; Choate and Yusef-Zadeh 1999), we investigated if there were any positional shifts between the peak mm and radio emission from Sgr A*. The detection of several stellar sources and the magnetar at both radio and mm wavelengths in the same epoch provides a means of registering the Galactic center at radio and near-IR frames. We calibrated the images astrometrically by using the common positions of sources listed in Table 1 . The positions in the near-IR images were measured via Gaussian fitting in AIPS. The astrometry was solved with the IDL solve-astro routine from ASTROLIB. No distortion solution was fitted, instead the linear terms were utilized, which involves slight shifts that exist between the 2 frames (VLT vs VLA). The VLT image was shifted in RA and Dec by 1.14 and 0.99 pixels with a pixel size of 29.033 milliarcseconds (mas). The rms scatter was 0.40 and 0.71 amongst the sources. While the VLA data should intrinsically have very good absolute pointing, the sources are so faint that the centroids of each source are fairly uncertain. The WCS coordinates of the VLT image were then modified to adjust for the shifts. The RA/Dec coordinates of the common sources were then computed which resulted in a better agreement to the VLA frame.

We included the uncertainty of the position of six stellar sources detected in both 226 (the first epoch) and $44 \mathrm{GHz}$ in the computation to register self-calibrated VLA and ALMA images. The pointing center of ALMA observations was set at $\alpha, \delta(J 2000)=17^{h} 45^{m} 40^{s} .040,-29^{\circ} 00^{\prime} 28^{\prime \prime} .2$. After calibrating the data using CASA and applying self-calibration gains, the peak position of Sgr A* is $\alpha, \delta(J 2000)=17^{h} 45^{m} 40^{s} .040004,-29^{\circ} 00^{\prime} 28^{\prime \prime} .1997$. The computed shifts that the $226 \mathrm{GHz}$ image needed to register radio and mm sources are -0.6524 and -5.821 pixels with 29.033 mas pixel $^{-1}$. We obtained the astrometrically-corrected position of $\mathrm{Sgr} \mathrm{A} *$ at $226 \mathrm{GHz}$ and determined that it is $3.37 \pm 0.04$ mas to the east and $11.03 \pm 0.23$ mas to the north of the radio position, thus the centroid of Sgr $\mathrm{A}^{*}$ at $226 \mathrm{GHz}$ is shifted by $11.53 \pm 0.23$ mas $\mathrm{NE}$ of the radio position at $44 \mathrm{GHz}$. This positional shift estimate assumes that the sources of ionized winds are symmetrical at 44 and $226 \mathrm{GHz}$, thus there is no optical depth effect that can be significant to explain the appearance of a shift in the position of Sgr A*.

Given that interstellar scattering is much smaller at $226 \mathrm{GHz}$ than at $44 \mathrm{GHz}$, the origin of the positional shift between radio and $\mathrm{mm}$ is not clear but it is likely that $\mathrm{Sgr} \mathrm{A}^{*}$ is contaminated by a strong mm source that shifts the bright position of Sgr A*. The source is resolved in Table 2 based on our second observation on 2016, July 18. The deconvolved angular size from our first epoch observation 2016, July 12 is $0.015^{\prime \prime} \times 0.013^{\prime \prime}\left(\mathrm{PA}=122 \pm 30^{\circ}\right)$. If the linear ridge in Figure 2b becomes brighter close to the peak of Sgr $\mathrm{A}^{*}$ at $226 \mathrm{GHz}$, it might be responsible for the shift. If so, this jet-like linear ridge arising from Sgr A* must have a hard or highly inverted spectrum since there is no significant emission detected at lower frequencies. In fact, recent high resolution 86 and 230 $\mathrm{GHz}$ observations of Sgr A* with milliarsecond resolutions show an asymmetric source structure (Brinkerink et al. 2016; Fish et al. 2016). This secondary component has a position angle PA $\sim 90^{\circ}$ and is shifted $100 \mu$ as to the east of the main source. This asymmetry could be explained by interstellar scattering effects or intrinsic to the source (Brinkerink et al. 2016). The positional shifts in these and VLBA measurements with vastly different spatial resolution suggests that they may be physically associated with each other and that the asymmetric source structure of Sgr A* is likely to be an intrinsic jet-like source emitted in the east-west direction before gets redirected to the northeast.

\subsection{Radial Fibrils of mm Emission}

One of the striking features we note in the mm images of the Galactic center are faint linear features with an extent ranging between 2 and $10^{\prime \prime}$. The widths of these narrow features, which we call fibrils, are unresolved spatially and their typical intensity is $\sim 50-100 \mu \mathrm{Jy}_{\text {beam }}{ }^{-1}$ above the background. Figure 7 a points to seven fibrils that are tentatively detected mainly to the NW and SW of Sgr A* at $226 \mathrm{GHz}$. Figures 7b with a slightly lower spatial resolution shows the innermost region of Figure $7 \mathrm{a}$ where radial fibrils are mainly found in the direction away from the Galactic center. Although weak linear features in complex radio images of Sgr A* can be problematic, it is difficult to see how the linear features in ALMA images could be artifacts. This is because of the geometry of the array which is has a spiral pattern unlike the linear configuration of the VLA. We also note that some of the fibrils appear to terminate at compact sources identified as radio and mm mass-losing young star to the SW of Sgr A*. Figure 8a shows a blow-up image of the region in reverse color where a network of fibrils with strongest emission is detected. The lines $a$ to $d$ are drawn parallel to the PA of individual fibrils. The best example of these 
faint sources witha varying background is $b$. Fibrils $c$ and $d$ appear to arise from stellar windy sources AF and AFNW. We also compared the mean value of the region where fibrils are detected in the region immediately to the east where there is no evidence of fibrils; the comparison showed a six-time-increase in the mean flux density of the region where fibrils are detected but the rms value was $0.1 \mathrm{mJy}^{\mathrm{beam}}{ }^{-1}$ in both regions. Another indication of the fibrils, along the line $c$ in Figure 8a, can be seen in Figure 8b showing a linear feature in the direction away from $\mathrm{AF} / \mathrm{HH}$ with an extent of $20^{\prime \prime}$. In order to bring out the weak and extended tail behind the AF star, the brightness of the mini-spiral is saturated. A schematic diagram of Figure 9 shows the faint fibrils as well as the dust and molecular layers (in black) associated with the mini-spiral. Given these caveats, sensitive measurements are clearly needed to confirm the tentative detection of fibrils described here.

\section{DISCUSSION}

\subsection{Low Extinction Millimeter Halo}

Chandra observations have characterized the X-ray emission surrounding Sgr A* as spatially extended with a radius of 1.5" (Baganoff et al. 2003; Wang et al. 2013; Rozanska et al. 2015). The X-ray luminosity is interpreted in terms of a radiatively inefficient accretion flow (RIAF, e.g. Yuan et al. 2004; Moscibrokzka et al. 2009). In this model, a fraction of the gaseous material accretes onto Sgr $\mathrm{A}^{*}$ and the rest is driven as an outflow from Sgr A* (e.g., Quataert 2004; Shcherbakov and Baganoff 2010; Wang et al. 2013). Alternatively, the diffuse X-ray emission associated with Sgr A* is interpreted as an expanding hot wind produced by the mass-loss from B-type main sequence stars, and/or the disks of photoevaporation of low mass young stellar objects (YSOs) at a rate $\sim 10^{-6} M_{\odot} \mathrm{yr}^{-1}$ (Yusef-Zadeh et al. 2016). The new millimeter halo emission and a dust cavity provide additional constraints on the origin of the gas in the inner $1^{\prime \prime}$ of Sgr A*.

The millimeter halo is coincident with the X-ray emission around Sgr A*, which is dominated by bremsstrahlung arising from a medium with $n_{e} \sim 150 \mathrm{~cm}^{-3}$ and $T \sim 3 \times 10^{7} \mathrm{~K}$. The bremsstrahlung contribution at $230 \mathrm{GHz}$, about $0.2 \mu \mathrm{Jy}$, is negligible. Thermal continuum from dust can also be ruled out because of the halo's extinction deficit of 0.5 magnitudes at $\mathrm{H}$-band relative to its surroundings. The millimeter emission could, however, be produced by synchrotron emission from relativistic electrons in equipartition with a $\sim 1.5 \mathrm{mG}$ magnetic field. The energy density of each of these components would then be $\sim 10 \%$ of the thermal energy density of the hot gas, so this is plausible. The luminosity in the $\mathrm{mm}$ is $4 \pi d^{2} v S_{v} \sim 1.4 \times 10^{33} \mathrm{erg} \mathrm{s}^{-1}$, comparable to the X-ray luminosity, $L_{X} \sim 1 \times 10^{33} \mathrm{erg} \mathrm{s}^{-1}$, implying that synchrotron cooling is marginally the dominant cooling mechanism for the gas. The synchrotron cooling time is $\sim 1000 \mathrm{yr}$, cf. the hot gas cooling time $\sim 10^{5} \mathrm{yr}$, so this requires electron acceleration on this time scale.

The coexistence of synchrotron emission with the hot gas supports a scenario in which the gas is produced by the interaction of winds either from the S-stars or by the photo-evaporation of low-mass YSO disks (Yusef-Zadeh at al 2016). In this picture the high relative speed of the orbital motion of the sources means that the gas is shocked to $\mathrm{keV}$ temperatures even though the outflow velocity from the sources is low. These shocks would also accelerate relativistic electrons. In steady state, the rate of conversion of kinetic energy to heat and relativistic electrons in shocks should equate to the X-ray and mm luminosities, respectively, therefore they should be approximately equal.

An alternative scenario is that outflow from the vicinity of Sgr $\mathrm{A}^{*}$ has created an X-ray/mm bubble in a denser medium. The extinction deficit $A_{K} \approx 0.5$ associated with the bubble is equivalent to a "missing" column density $\sim 10^{22} \mathrm{~cm}^{-2}$, or a number density $n_{H} \sim 3 \times 10^{4} \mathrm{~cm}^{-3}$. The current pressure inside the bubble would drive expansion at a speed of $\sim 40 \mathrm{~km} \mathrm{~s}^{-1}$ into such a medium, yielding an expansion time scale of $\sim 1300 \mathrm{yr}$.

One possible origin of the outflow is from mass-losing evolved and/or young stars. Because of the inverted spectrum of mass-losing stars the emission is stronger at at higher frequencies. Assuming a typical flux density $\sim 1 \mathrm{mJy}$ at $226 \mathrm{GHz}$, a total of 80 stars are needed to account for the diffuse emission. So, in this picture, the diffuse millimeter emission should resolve into individual stars with stellar winds. Because of the high orbital motion of stars and higher mass loss rates, $\sim 10^{-7}-10^{-6} M_{\odot} \mathrm{yr}^{-1}$ from recent modeling (Offner \& Arce 2015), the X-ray gas is supplied by shocked winds. Similar to mm emission, X-ray emission should be resolved into individual stellar sources. One possibility is enhanced X-ray emission at about $1.06^{\prime \prime}$ from the peak X-ray emission from Sgr A*. This emission appears to coincide with IRS 16C.

In summary, we have presented a variety of structures within $30^{\prime \prime}$ of $\mathrm{Sg} \mathrm{A}^{*}$ (see the diagram in Figure 9) using data taken with the VLA and ALMA. On the smallest scale, we detect $226 \mathrm{GHz}$ emission from a $2.5^{\prime \prime} \times 1.5^{\prime \prime}$ halo 
that appears to coincide with a dust cavity and diffuse X-ray gas centered on Sgr A*. This mm emission coincides with diffuse X-ray emission centered on Sgr A*. We argued that the mm emission is due to synchrotron, generated either from fast-moving orbiting stars or protostars or from the activity associated with Sgr A*. This implies an outflow that produced the $\mathrm{mm}$ and X-ray emission and destroyed dust grains. On a scale of $5^{\prime \prime}$ from Sgr A*, we detect elongated balloon-shaped structure and a dust cavity that are roughly in the direction where a number of head-tail radio sources are found in previous measurements. These morphological details can be described by a collimated outflow from Sgr $\mathrm{A}^{*}$ at a position angle of $60^{\circ}$. We also detected $\mathrm{mm}$ emission from ionized winds of massive stars orbiting Sgr $\mathrm{A}^{*}$ and determined their spectral indices. Lastly, we found a discrepancy in the peak position of Sgr A* between radio and $226 \mathrm{GHz}$. Future high frequency ALMA observations should be able to place a better constraint on frequency-depended position of Sgr A* and to confirm tentative detection of a network of faint fibrils distributed throughout the inner $15^{\prime \prime}$ of the Galactic center.

Acknowledgments: We thank the referee for excellent comments. This work is partially supported by the grant AST-0807400 from the NSF and the European Research Council under the European Union's Seventh Framework Program (FP/2007-2013). The research leading to these results has received funding from the European Research Council under the European Union's Seventh Framework Program (FP7/2007-2013) / ERC grant agreement number 614922] and by an Outside Studies Program Fellowship awarded to Macquarie University. The National Radio Astronomy Observatory is a facility of the National Science Foundation, operated under a cooperative agreement by Associated Universities, Inc.

\section{REFERENCES}

Baganoff, F. K., et al. 2003, ApJ, 591, 891

Boehle, A., Ghez, A. M., Schödel, R., et al. 2016, ApJ, 830, 17

Brinkerink, C. D., Müller, C., Falcke, H., et al. 2016, MNRAS, 462, 1382

Cotton, W. D. 2008, PASP, 120, 439

Eckart, A., Horrobin, M., Britzen, S., et al. 2014, The Galactic Center: Feeding and Feedback in a Normal Galactic Nucleus, 303,269

Eckart, A., Mu Vzić, K., Yazici, S., et al. 2013, A\&A, 551, A18

Fish, V. L., Johnson, M. D., Doeleman, S. S., et al. 2016, ApJ, 820, 90

Genzel, R., Eisenhauer, F., \& Gillessen, S. 2010, Reviews of Modern Physics, 82, 3121

Gillessen, S., Eisenhauer, F., Fritz, T. K., et al. 2009, ApJ, 707, L114

Gillessen, S., Genzel, R., Fritz, T. K., et al. 2012, Nature, 481, 51

Gillessen, S., Plewa, P. M., Eisenhauer, F., et al. 2017, ApJ, 837, 30

Kennea, J.A., Burrows, D.N., Kouveliotou, C., Palmer, D.M., et al. 2013, ApJ, 770, L24

Lu, J. R., Ghez, A. M., Hornstein, S. D., et al. 2009, ApJ, 690, 1463

Markoff, S., Bower, G. C., \& Falcke, H. 2007, MNRAS, 379, 1519

Mu Vzić, K., Eckart, A., Schödel, R., et al. 2010, A\&A, 521, A13

Mu Vzić, K., Eckart, A., Schödel, R., Meyer, L., \& Zensus, A. 2007, A\&A, 469, 993

Offner, S. S. R., \& Arce, H. G. 2015, ApJ, 811, 146

Panagia, N. 1973, AJ, 78, 929

Panagia, N., \& Felli, M. 1975, A\&A, 39, 1

Paumard, T., Genzel, R., Martins, F., Nayakshin, S., Beloborodov et al. 2006, ApJ, 643, 1011

Pfuhl, O., Gillessen, S., Eisenhauer, F., et al. 2015, ApJ, 798, 111

Pott, J.-U., Eckart, A., Glindemann, A., et al. 2008, A\&A, 480, 115

Quataert, E. 2004, ApJ, 613, 322

Reid, M. J., \& Brunthaler, A. 2004, ApJ, 616, 872

Reid, M. J., Readhead, A. C. S., Vermeulen, R. C., \& Treuhaft, R. N. 1999, ApJ, 524, 816

Różańska, A., Mróz, P., Mościbrodzka, M., Sobolewska, M., \& Adhikari, T. P. 2015, A\&A, 581, A64

Shcherbakov, R. V., \& Baganoff, F. K. 2010, ApJ, 716, 504

Schödel, R., Najarro, F., Muzic, K., \& Eckart, A. 2010, A\&A, 511, A18

Schödel, R., Eckart, A., Alexander, T., et al. 2007, A\&A, 469, 125

Schödel, R., Feldmeier, A., Kunneriath, D., et al. 2014, A\&A, 566, A47

Shannon, R. M., \& Johnston, S. 2013, MNRAS, 435, L2

Torne, P., Desvignes, G., Eatough, R. P., et al. 2017, MNRAS, 465, 242

Valencia-Schneider et al. 2015, ApJ, 800, 125

Viehmann, T., Eckart, A., Schödel, R., et al. 2005, A\&A, 433, 117

Wang, Q. D., Nowak, M. A., Markoff, S. B., et al. 2013, Science, 341, 981

Wardle, M., \& Yusef-Zadeh, F. 1992, Nature, 357, 308

Witzel, G., Ghez, A. M., Morris, M. R., et al. 2014, ApJ, 796, LL8

Yelda, S., Ghez, A. M., Lu, J. R., et al. 2014, ApJ, 783, 131

Yelda, S., Lu, J. R., Ghez, A. M., et al. 2010, ApJ, 725, 331

Yuan, F., Quataert, E. \& Narayan, R. 2004, ApJ, 606, 894

Yusef-Zadeh, F., Diesing, R., Wardle, M., et al. 2015, ApJ, 811, L35 
Yusef-Zadeh, F., Wardle, M., Sewilo, M., et al. 2015, ApJ, 808, 97 Yusef-Zadeh, F., Bushouse, H., Schödel, R., et al. 2015, ApJ, 809, 10 Yusef-Zadeh, F., Wardle, M., Schödel, R., et al. 2016, ApJ, 819, 60

Yusef-Zadeh, F., Roberts, D., Heinke, C., et al. 2014b, The Astronomer's Telegram, 6041, 1

Yusef-Zadeh, F., Cotton, W., Wardle, M., et al. 2017, MNRAS,

Yusef-Zadeh, F., Arendt, R., Bushouse, H., et al. 2012, ApJ, 758, L11

Yusef-Zadeh, F., Roberts, D. A., Bushouse, H., et al. 2014, ApJ, 792, L1

Yusef-Zadeh, F., Choate, D., \& Cotton, W. 1999, ApJ, 518, L33

Yusef-Zadeh, F., Morris, M., \& Ekers, R. D. 1990, Nature, 348, 45

Zhao, J.-H., Goss, W. M., Lo, K. Y., \& Ekers, R. D. 1991, Nature, 354, 46

Zhao, J.-H., Morris, M. R., Goss, W. M., \& An, T. 2009, ApJ, 699, 186 
L10 F. Yusef-Zadeh

This paper has been typeset from a $\mathrm{TE}_{\mathrm{E}} \mathrm{X} / \mathrm{LA} \mathrm{EX}$ file prepared by the author. 

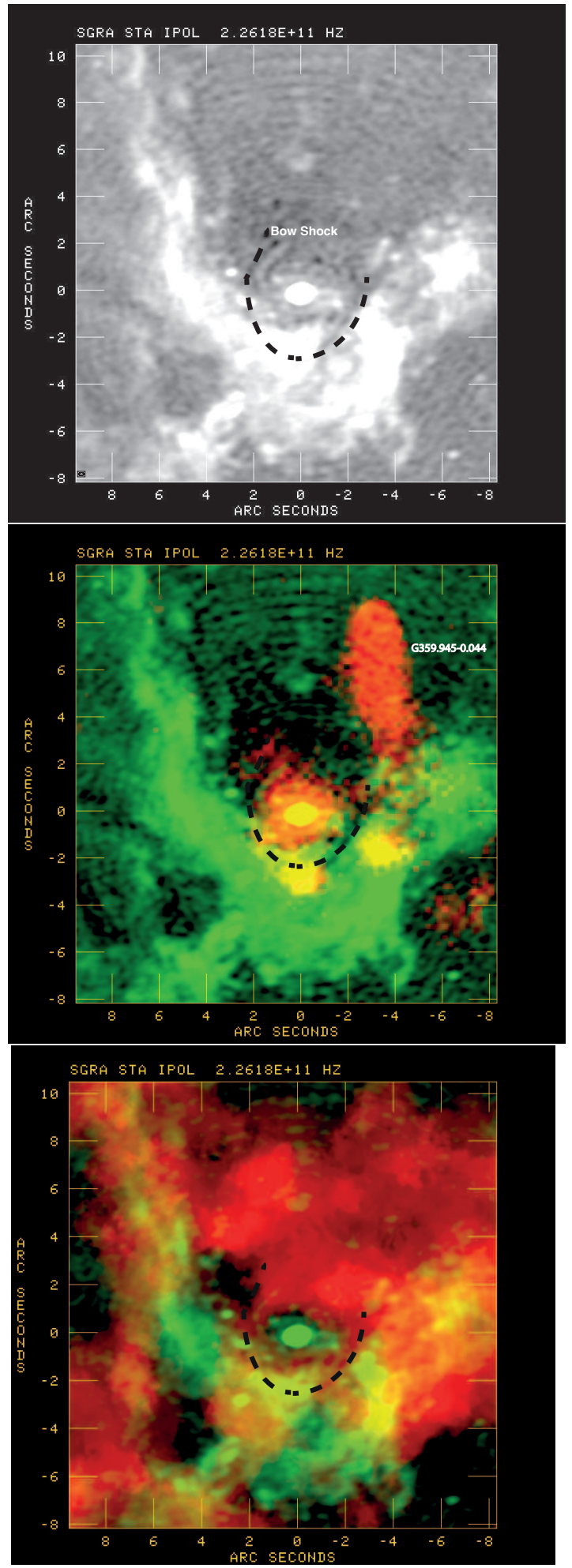

Figure 1. (a) A $226 \mathrm{GHz}$ image of the mini-spiral with spatial resolution of $0.38^{\prime \prime} \times 0.27^{\prime \prime}$ and $\mathrm{PA}=-79^{\circ}$. This image is based on combining both observations taken in two epochs on 2016, July 12 and 18. The peak flux is $3.47 \mathrm{Jy} \mathrm{beam}^{-1}$. (The grayscale range $-6.7 \times 10^{-4}-3 \times 10^{-3} \mathrm{Jy} \mathrm{beam}^{-1}$.) (b) Similar to (a) except that an X-ray 1.5-7 keV image in red taken with Chandra (D. Haggard, private communication) is superimposed on a $226 \mathrm{GHz}$ image in green. (The grayscale range 0 to $10^{4}$ counts.) $-6 \times 10^{-4}-3 \times 10^{-3} \mathrm{Jy}_{\text {beam }}{ }^{-1}$.) (c) Similar to (a) except the Ks extinction image (Schödel et al. 2010) in red is superimposed on a $226 \mathrm{GHz}$ image in green. The range of extinction value is between 2.35 and 3.20 magnitudes. (The grayscale range $-6.7 \times 10^{-4}-1 \times 10^{-2} \mathrm{Jy}_{\text {beam }}{ }^{-1}$.) 

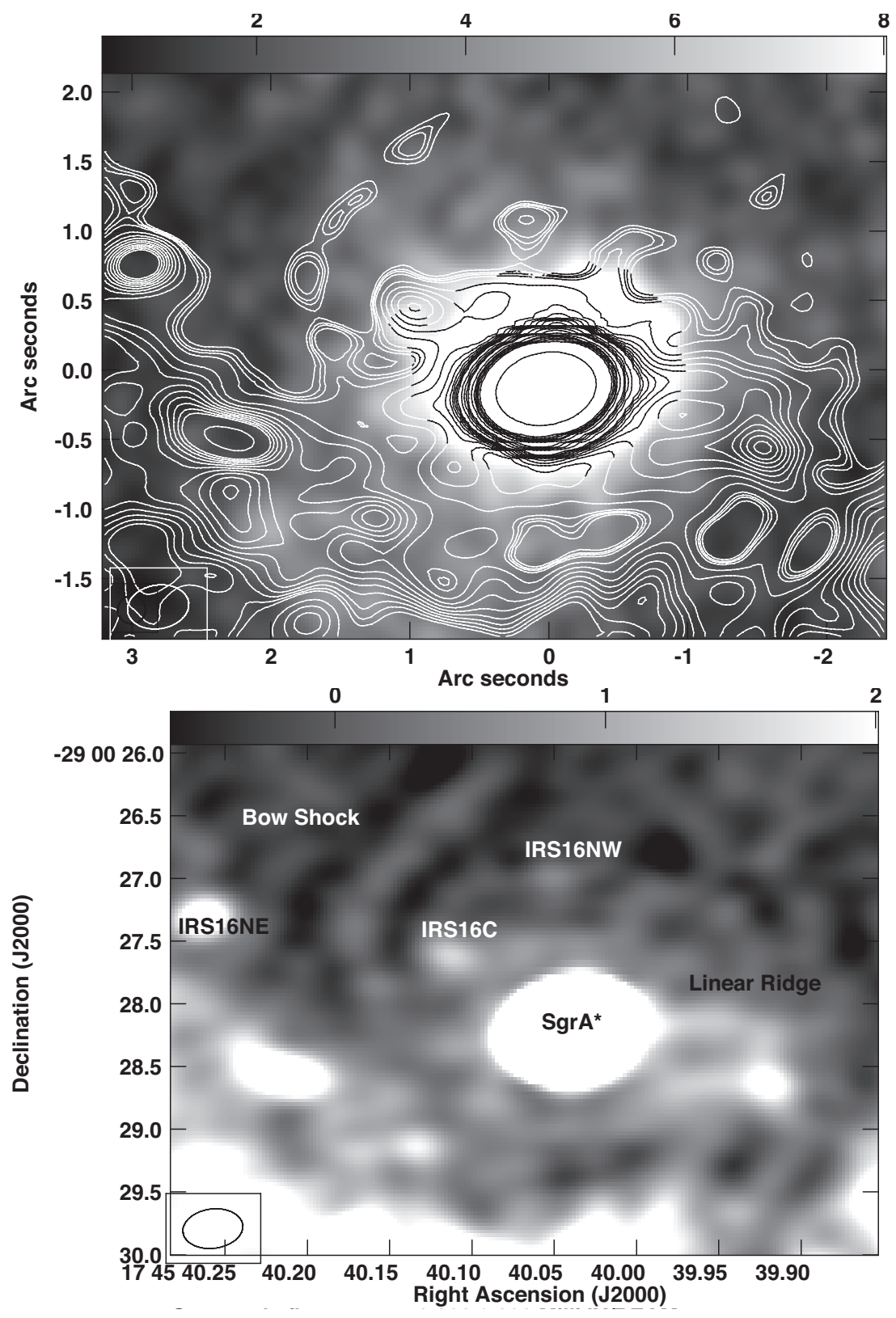

Figure 2. (a) Contours of $226 \mathrm{GHz}$ emission with levels set at $(1,2, \ldots, 10,12,15,20,25,30,35,40,50,55,60,65,70$, $80,90,110,130) \times 0.15 \mathrm{mJy}_{\text {beam }}{ }^{-1}$ are superimposed on an 1.5-7 keV X-ray image of Sgr A* with the spatial resolution of $\sim 0.5^{\prime \prime}$. (The grayscale range 0 to $8 \times 10^{3}$ counts.) (b) A grayscale image of the mm emission from the inner $4^{\prime \prime} \times 6^{\prime \prime}$ of Sgr $\mathrm{A}^{*}$ with similar resolution to the $226 \mathrm{GHz}$ image in (a). Prominent stellar sources, the bow shock and the linear features are labeled. (The grayscale range $-1 \times 10^{-3}-3 \times 10^{-3} \mathrm{Jy} \mathrm{beam}^{-1}$.) 

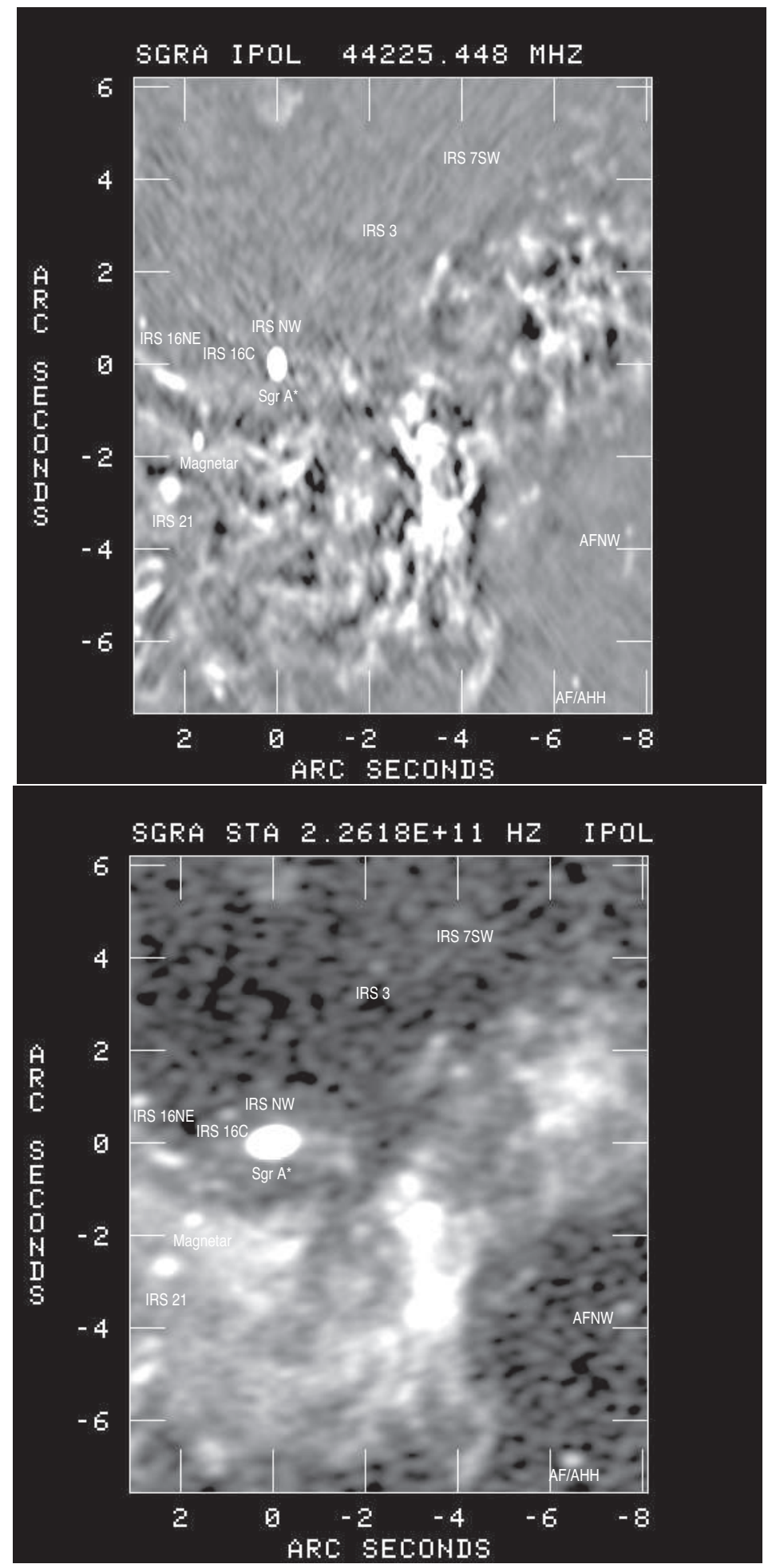

Figure 3. (a) Top A $44 \mathrm{GHz}$ image of the mini-spiral is constructed by limiting the $u v$ range to greater than $100 \mathrm{k} \lambda$ with a spatial resolution of $0.22^{\prime \prime} \times 0.13^{\prime \prime}\left(\mathrm{PA}=3.8^{\circ}\right)$ from the 2016 , July 12 epoch. (The grayscale range $-5 \times 10^{-4}-5 \times 10^{-4} \mathrm{Jy}$ beam $^{-1}$.) (b) Bottom A $226 \mathrm{GHz}$ image of the mini-spiral with similar resolution to that of Figure 1a. Labeled sources are compact with properties listed in Tables 1 and 2. (The grayscale range $-6.7 \times 10^{-4}-1 \times 10^{-2} \mathrm{Jy} \mathrm{beam}^{-1}$.) 

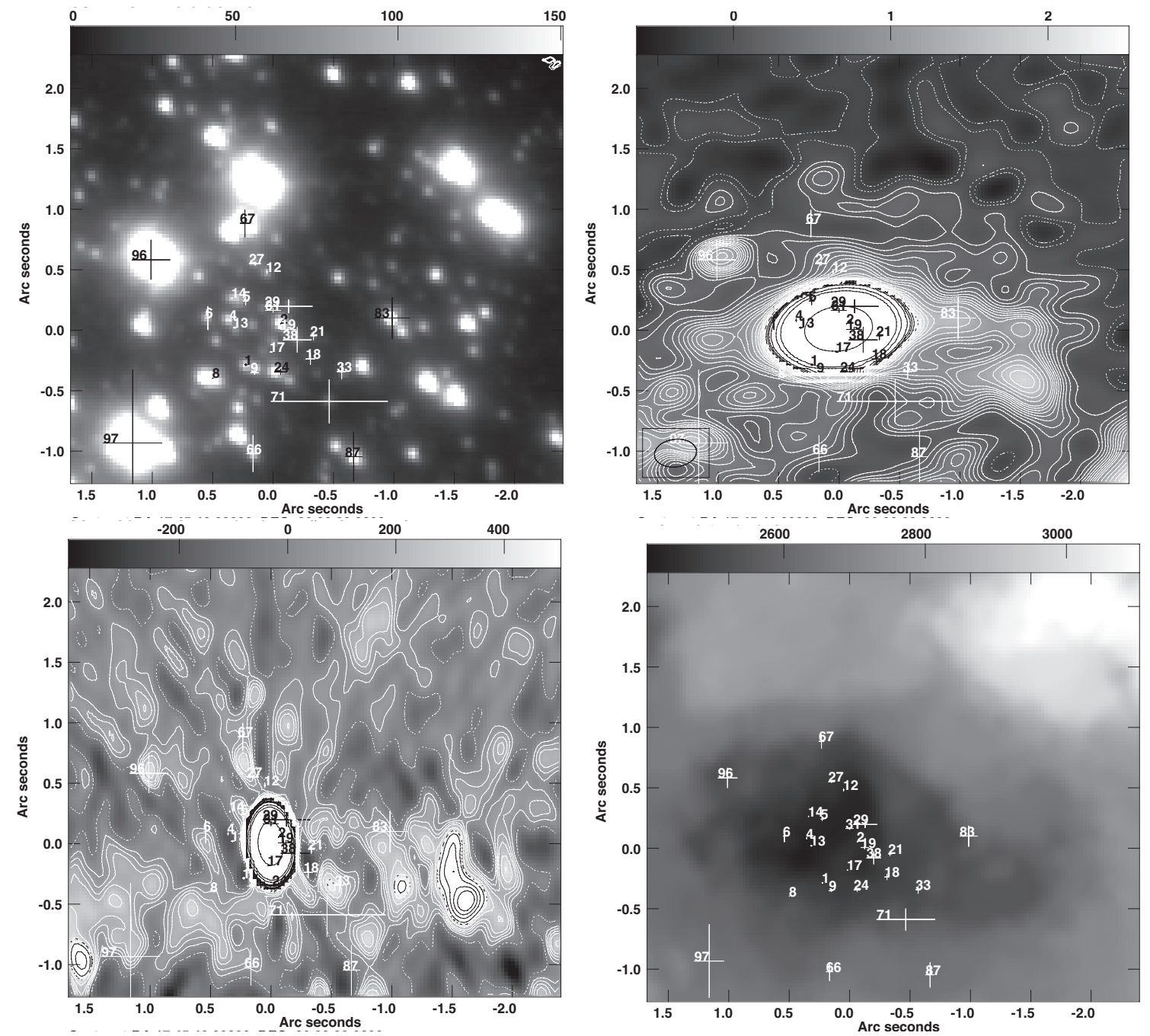

Figure 4. (a) Top Left A grayscale image of the inner 3.5" of Sgr A* at $\mathrm{H}$ band taken on 2016, July 12. (b) Top Right Grayscale contours of $226 \mathrm{GHz}$ emission set at $(-2,-1,1,2, \ldots, 10,12, \ldots, 20,25,30,35,40,50,100,200,5000) \times 0.1 \mathrm{mJy}^{-1}$ beam $^{-1}$ with a spatial resolution of $0.35^{\prime \prime} \times 0.23^{\prime \prime}\left(\mathrm{PA}=-82.7^{\circ}\right)$. (c) Bottom Left Grayscale contours of $44.2 \mathrm{GHz}$ emission set at $(-2,-1,1,2, \ldots, 10,12, . .20,25,30,35,40,50,100,200,5000) \times 0.05 \mathrm{mJy}$ beam $^{-1}$ with a spatial resolution of $0.22^{\prime \prime} \times 0.13^{\prime \prime}$ $\left(\mathrm{PA}=3.8^{\circ}\right)$, taken on 2016, July 12. (d) Bottom Right An extinction map showing an elongated dust cavity coincident with the S cluster (Schoödel et al. 2009). Stellar sources associated with the S cluster are labeled at their expected positions on 2016, July 12. The extinction ranges between 2.4 and 3.14 magnitudes. 

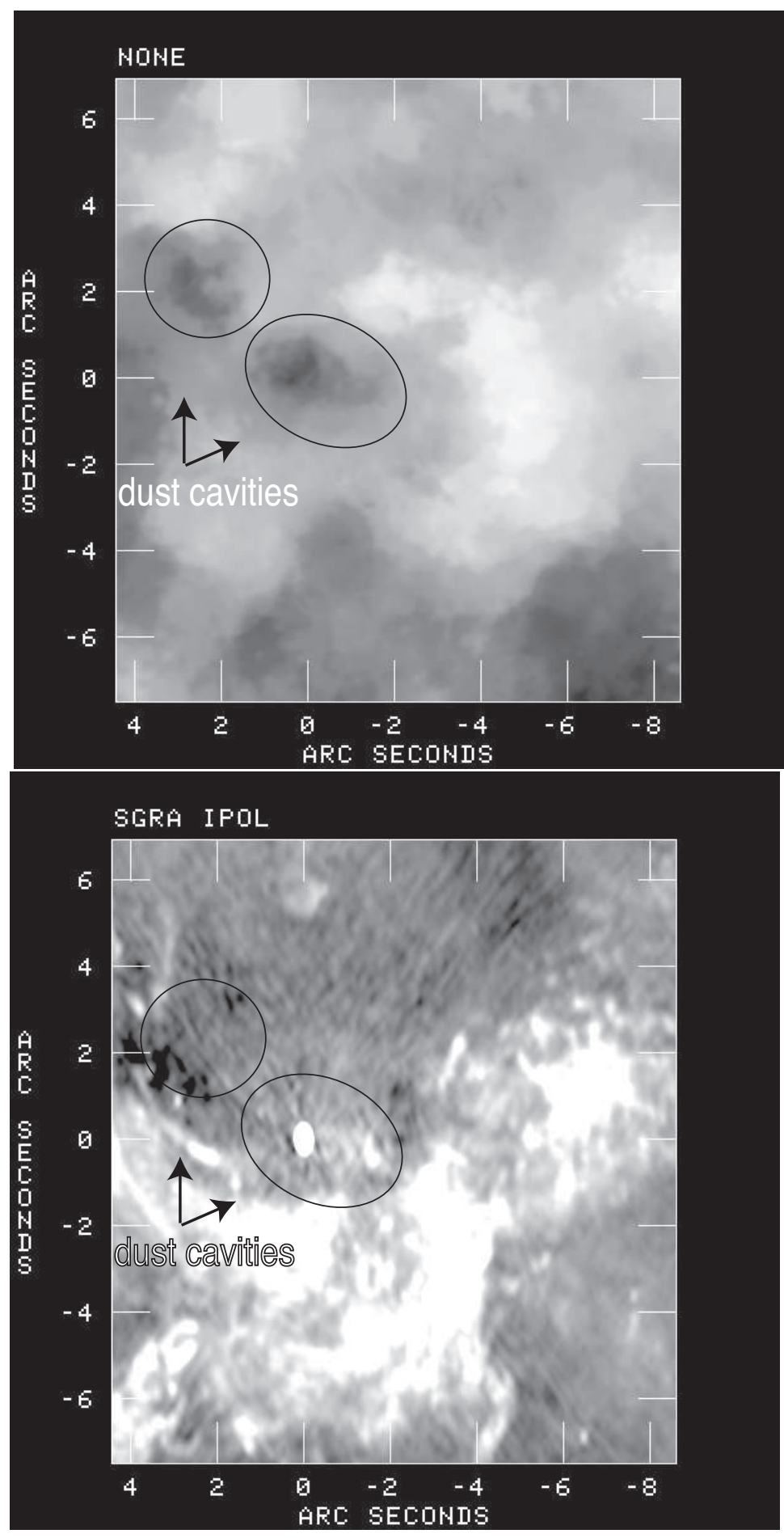

Figure 5. (a) Top Similar to the region shown in Figure $4 \mathrm{~d}$ except the inner $13^{\prime \prime} \times 13^{\prime \prime}$ of Sgr A*. The extinction ranges between 2.4 and 3.20 magnitudes. (b) Bottom Similar to the region shown in Figure 3a except that $u v$ data was not truncated, resulting a spatial resolution of $0.24^{\prime \prime} \times 0.14^{\prime \prime}\left(\mathrm{PA}=3.3^{\circ}\right)$. (The grayscale range $-3 \times 10^{-4}-1 \times 10^{-3} \mathrm{Jy} \mathrm{beam}^{-1}$.) 


\section{SGRA IFOL}
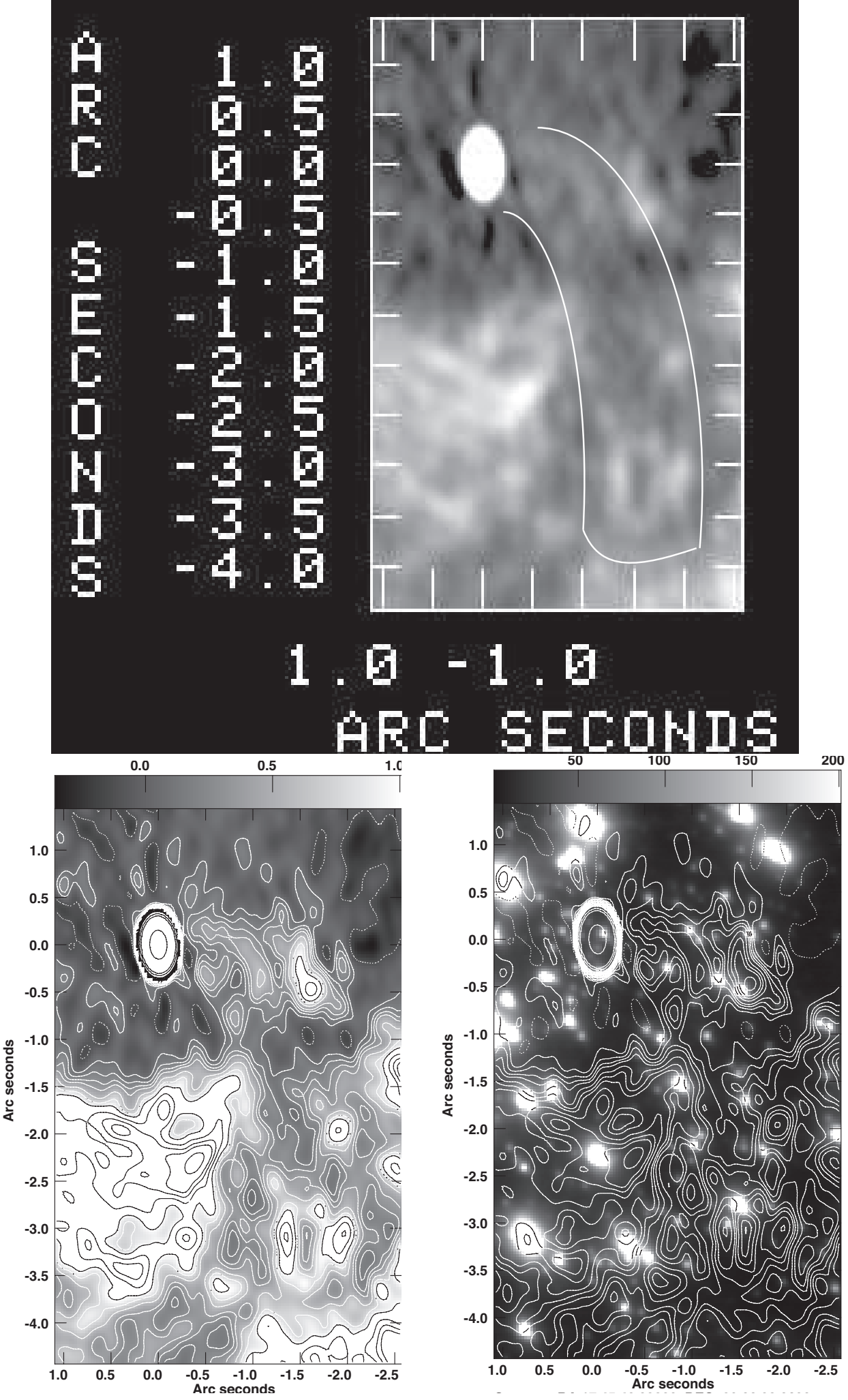

Figure 6. (a) Top Similar to Figure $5 \mathrm{~b}$ except the inner $3.5^{\prime \prime} \times 6^{\prime \prime}$ of Sgr A*. (b) Bottom Left Similar to (a) except showing grayscale contours of emission set at $(-2,-1,1,2, \ldots, 10,14,18,22,30,38,46,100,200,5000) \times 0.1$ mJy beam $^{-1}$. (c) Bottom Right Similar to (b) except that the $44 \mathrm{GHz}$ contours are superimposed on a H-band image. 


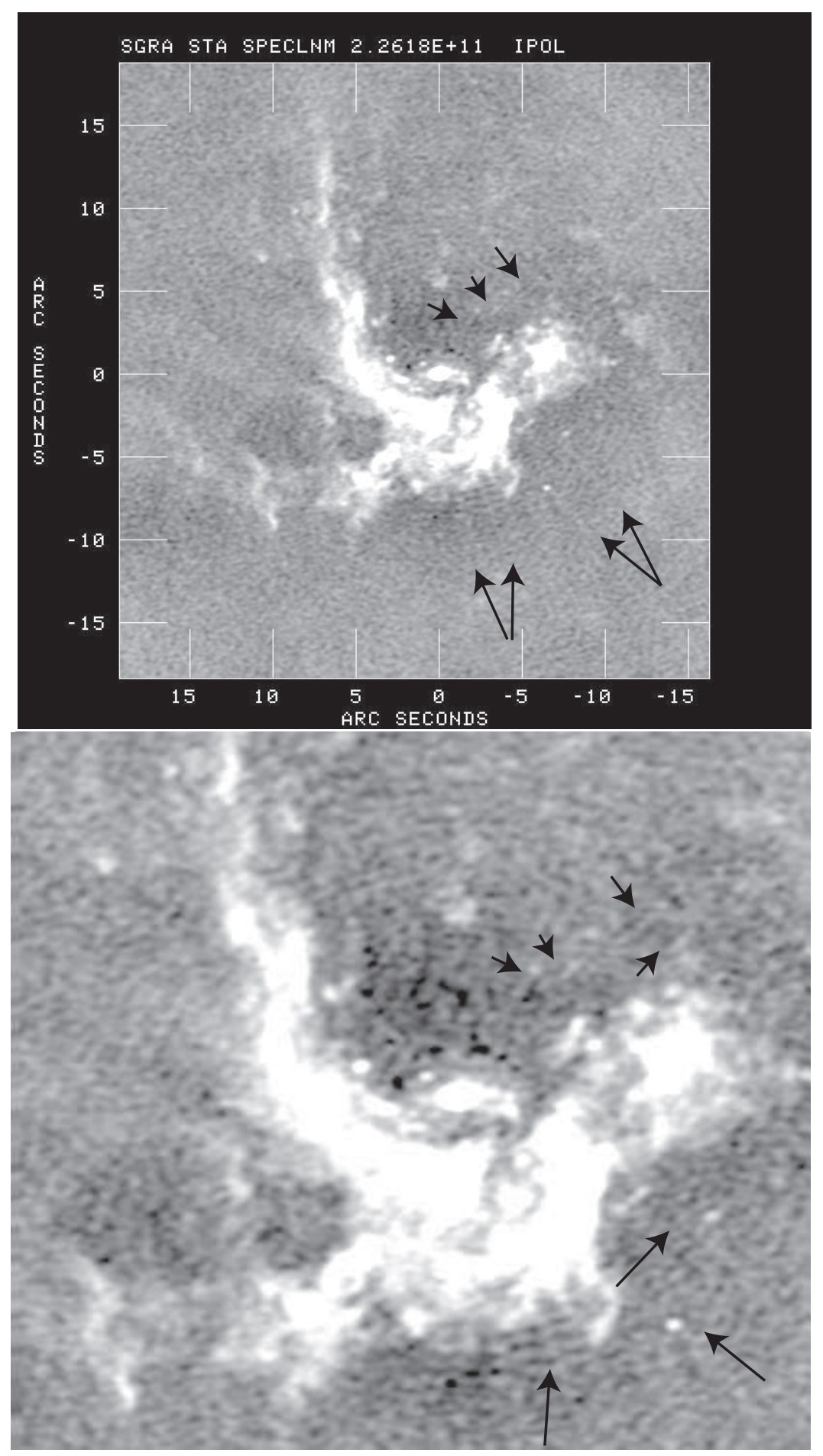

Figure 7. (a) Top A Grayscale $226 \mathrm{GHz}$ image of the mini-spiral with a spatial resolution of $0.22^{\prime \prime} \times 0.13^{\prime \prime}\left(\mathrm{PA}=3.8^{\circ}\right)$ taken on 2016, July 18 with ALMA. (b) Bottom The inner quarter of the $226 \mathrm{GHz}$ image shown in (a) with a resolution of $0.36^{\prime \prime} \times 0.24^{\prime \prime}\left(\mathrm{PA}=-82^{\circ} .4\right.$. The arrows point to faint fibrils detected in radio and mm images. 


\section{L18 F. Yusef-Zadeh}
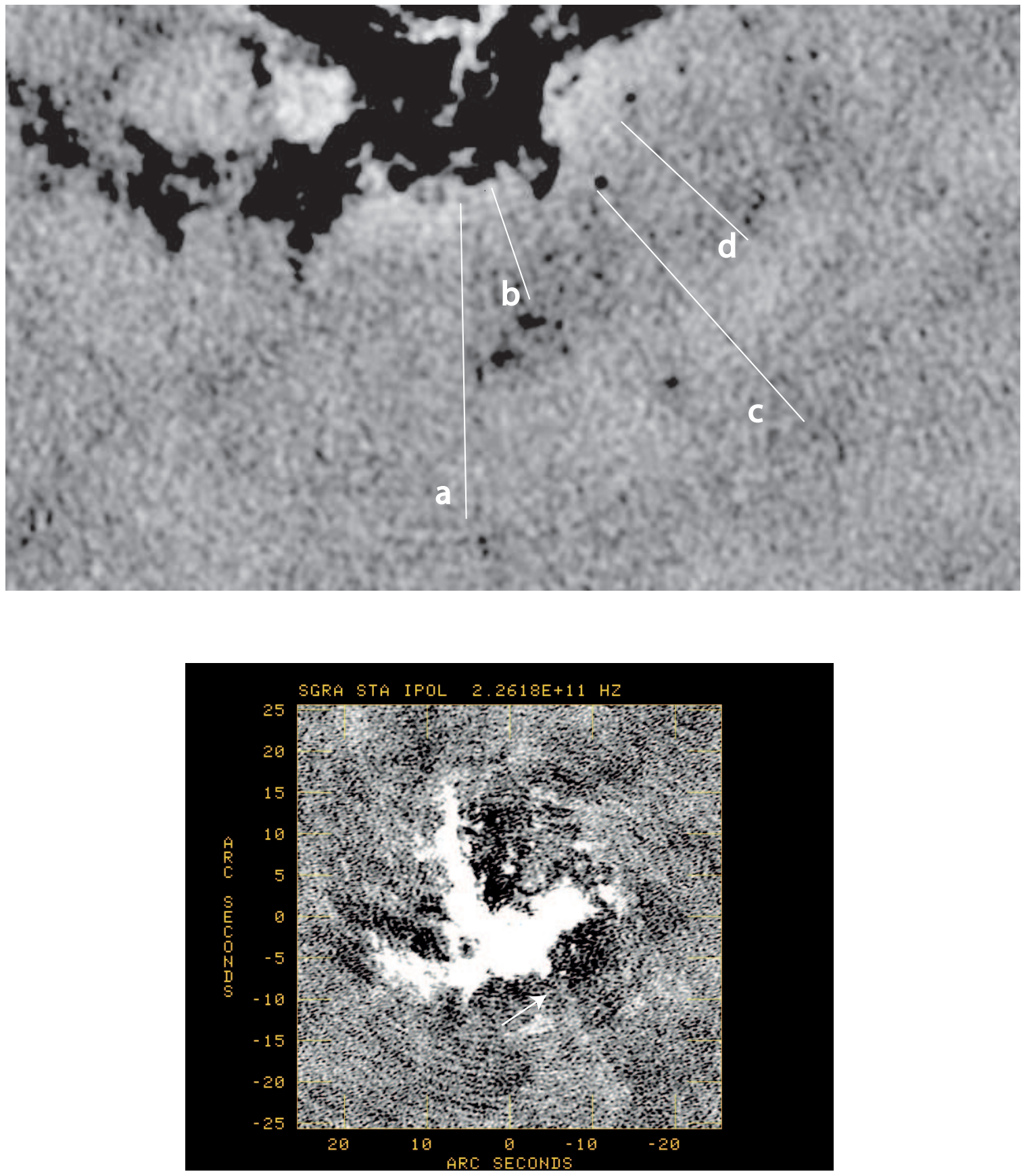

Figure 8. (a) Top A $226 \mathrm{GHz}$ image (in reverse color) with a resolution of $0.45^{\prime \prime} \times 0.45^{\prime \prime}$ from the 2016, July 18 data taken with ALMA. The drawn paralled lines point where the fibrils are located. (b) Bottom A $226 \mathrm{GHz}$ image with a spatial resolution of $0.44^{\prime \prime} \times 0.34^{\prime \prime}\left(\mathrm{PA}=-70.8^{\circ}\right)$ taken on 2016, July 12 with ALMA. (The grayscale range $-3 \times 10^{-4}-5 \times 10^{-4} \mathrm{Jy}$ beam $^{-1}$.) 


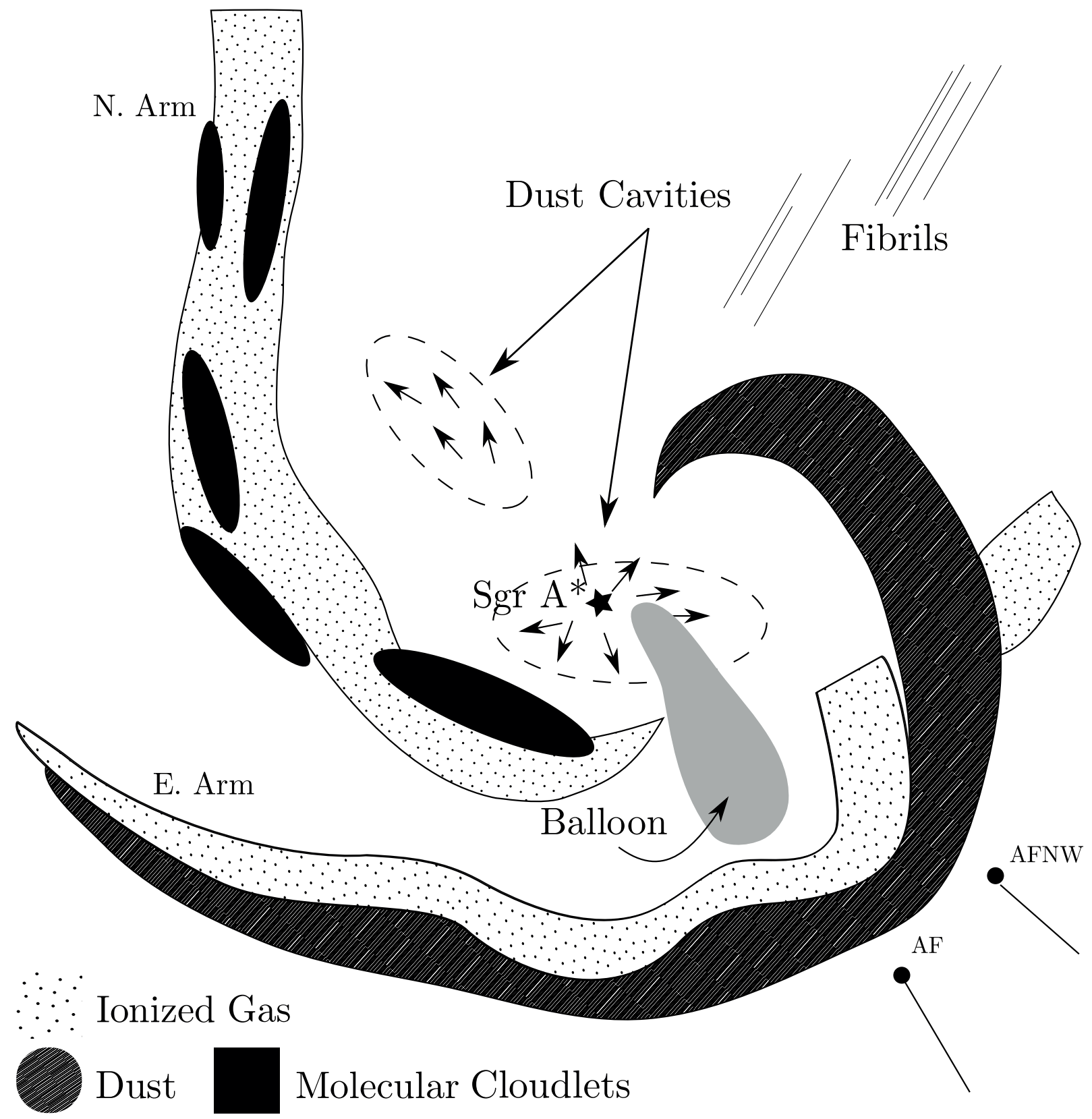

Figure 9. A schematic picture of major features found at $226 \mathrm{GHz}$. Dark features point to regions where extended dust and neutral gas is detected. 
Table 1. Parameters of 2D Gaussian fits to $44 \mathrm{GHz}$ Stellar Sources$$
\text { RA (J2000) Dec }(\mathrm{J} 2000)
$$

Distance

Position

$\theta_{a} \times \theta_{b}(\mathrm{PA})$

Peak Intensity

(arcsec) Accuracy

Sgr A*
IRS $16 \mathrm{C}$

IRS $16 \mathrm{C}$

$\left(17^{\mathrm{h}} 45^{\mathrm{m}}\right)$

$\left(-29^{\circ} 00^{\prime}\right)$

$(\operatorname{arcsec})$

(mas)
0.00

mas $\times$ mas (deg)

40.1134

28.0690

27.4370
26.8459

12.24

$\times 0(31)$

$193 \times 155(177)$
$109 \times 0(149)$

$\left(\mathrm{mJy} \mathrm{beam}^{-1}\right)$

Magnetar $\quad 40.1685$

IRS $16 \mathrm{NE} \quad 40.2608$

29.7421

IRS2

40.2154

IRS 3

IRS 7SW

39.8614

39.7383

AFNW

39.4558

39.5446

30.7693

24.2147

23.2063

31.6992

34.9672

1.17

16.41

$74 \times 0(157)$

$100 \times 0(13)$

$1.63 \quad 258 \times 236(98) \quad 4.049 \pm 0.048$

$0.502 \pm 0.049$

$0.317 \pm 0.052$

$5.787 \pm 0.052$

$0.977 \pm 0.052$

$50.24 \quad 181 \times 0(139)$

$63.47250 \times 0(16)$

$10 \mathrm{AF} / \mathrm{AHH}$

$4.049 \pm 0.048$

$0.109 \pm 0.052$

$0.111 \pm 0.051$
$0.411 \pm 0.052$

$0.735 \pm 0.052$

Spectral Index

Integrated Flux

$(\alpha)$

$0.56 \pm 0.00004 \quad 1536.900 \pm 0.090$

$0.67 \pm 0.29 \quad 1.044 \pm 0.143$

$\begin{array}{ll}0.28 \pm 0.81 & 0.293 \pm 0.085\end{array}$

$-0.21 \pm 0.10 \quad 5.866 \pm 0.091$

$0.79 \pm 0.12 \quad 1.056 \pm 0.095$

$0.23 \pm 0.07-13.309 \pm 0.199$

$1.14 \pm 0.66-0.121 \pm 0.096$

$1.12 \pm 0.63=0.147 \pm 0.107$

$\begin{array}{ll}1.12 \pm 0.63 & 0.147 \pm 0.107 \\ 0.73 \pm 0.31 & 0.488 \pm 0.100\end{array}$

$0.84 \pm 0.15 \quad 0.717 \pm 0.088$ 
Table 2. Parameters of 2D Gaussian fits to $226 \mathrm{GHz}$ Stellar Sources

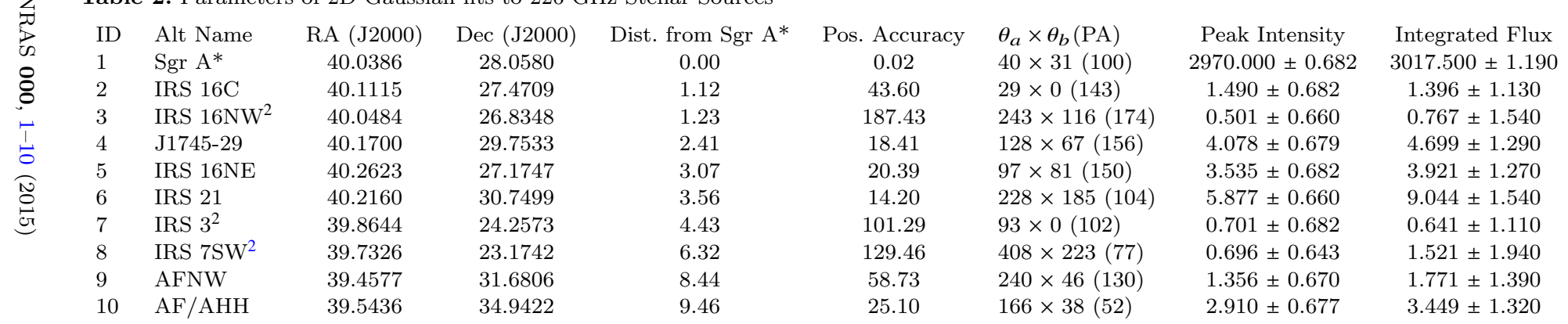


Table 3. Predicted Positions of Young Stars in the S Cluster from Sgr A* at the 2016.54 Epoch

$\begin{array}{lcccc}\text { Source Name } & \begin{array}{c}\text { RA (offset) } \\ \text { arcsec }\end{array} & \begin{array}{c}\text { Dec (offset) } \\ \text { arcsec }\end{array} & \begin{array}{c}\left(\sigma_{X}\right) \\ \text { arcsec }\end{array} & \begin{array}{c}\left(\sigma_{Y}\right) \\ \text { arcsec }\end{array} \\ \text { S1 } & 0.2244 & -0.2862 & 0.0161 & 0.0187 \\ \text { S2 } & -0.0632 & 0.0536 & 0.0012 & 0.0038 \\ \text { S4 } & 0.3550 & 0.0826 & 0.0226 & 0.0103 \\ \text { S5 } & 0.2259 & 0.2417 & 0.0622 & 0.0429 \\ \text { S6 } & 0.5399 & 0.0996 & 0.1895 & 0.0350 \\ \text { S8 } & 0.4982 & -0.3972 & 0.0048 & 0.0063 \\ \text { S9 } & 0.1531 & -0.3513 & 0.0272 & 0.0623 \\ \text { S12 } & 0.0495 & 0.4826 & 0.0385 & 0.0125 \\ \text { S13 } & 0.3160 & 0.0198 & 0.0128 & 0.0273 \\ \text { S14 } & 0.3391 & 0.2641 & 0.0132 & 0.0103 \\ \text { S17 } & 0.0161 & -0.1812 & 0.0040 & 0.0205 \\ \text { S18 } & -0.3088 & -0.2381 & 0.0932 & 0.0719 \\ \text { S19 } & -0.1253 & 0.0057 & 0.0381 & 0.1234 \\ \text { S21 } & -0.3348 & -0.0473 & 0.0644 & 0.0498 \\ \text { S24 } & -0.0634 & -0.3448 & 0.0472 & 0.1037 \\ \text { S27 } & 0.1491 & 0.5496 & 0.0256 & 0.0944 \\ \text { S29 } & -0.1297 & 0.1967 & 0.1094 & 0.3917 \\ \text { S31 } & -0.0000 & 0.1630 & 0.0340 & 0.1237 \\ \text { S33 } & -0.5679 & -0.3434 & 0.1219 & 0.0737 \\ \text { S38 } & -0.1996 & -0.0801 & 0.2040 & 0.2352 \\ \text { S66 } & 0.1655 & -1.0205 & 0.3000 & 0.1063 \\ \text { S67 } & 0.2320 & 0.8833 & 0.2265 & 0.0823 \\ \text { S71 } & -0.4660 & -0.5897 & 0.3592 & 0.9643 \\ \text { S83 } & -0.9859 & 0.0999 & 0.3428 & 0.2893 \\ \text { S87 } & -0.6667 & -1.0474 & 0.4086 & 0.1390 \\ \text { S96 } & 1.0095 & 0.5818 & 0.3255 & 0.3163 \\ \text { S97 } & 1.1609 & -0.9340 & 1.2096 & 0.4843\end{array}$

